\title{
RESEARCH ON UPPER-BODY EXOSKELETONS FOR Performance Augmentation of Production Workers
}

\author{
Mohamed Abdelmomen, ${ }^{1}$ Fuat Ozan Dengiz, ${ }^{2}$ Hoda Samir ${ }^{3} \&$ Mart Tamre ${ }^{4}$ \\ 1,2,3,4 Tallinn University of Technology, School of Engineering, Centre of Mechatronics and Autonomous Systems, Ehitajate tee 5, \\ 19086 Tallinn, Estonia \\ ${ }^{1}$ mabdel@ taltech.ee, ${ }^{2}$ fudeng@ taltech.ee, ${ }^{3}$ hosami@ taltech.ee, ${ }^{4}$ mart.tamre@ taltech.ee
}
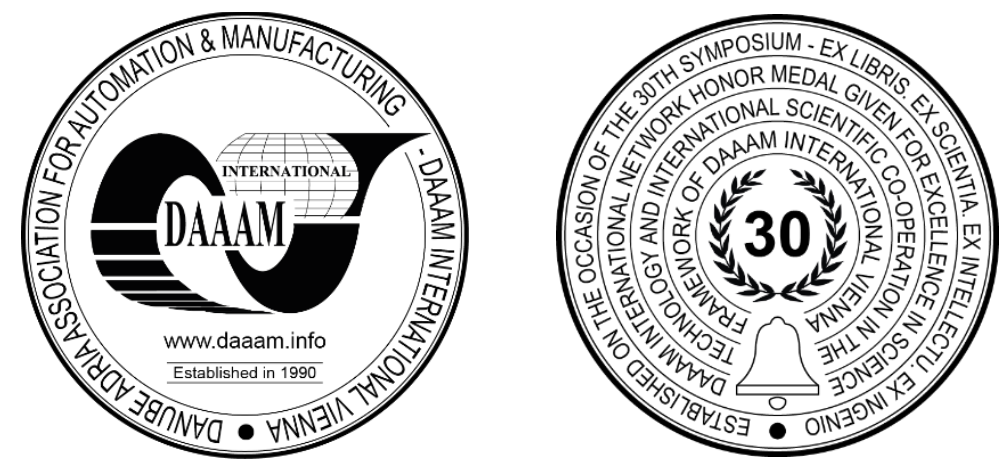

This Publication has to be referred as: Abdelmomen, M[ohamed]; Dengiz, O[zan]; Hoda, S[amir] \& Tamre, M[art] (2019). Research on Upper-Body Exoskeletons for Performance Augmentation of Production Workers, Proceedings of the 30th DAAAM International Symposium, pp.0904-0913, B. Katalinic (Ed.), Published by DAAAM International, ISBN 978-3-902734-22-8, ISSN 1726-9679, Vienna, Austria

DOI: $10.2507 / 30$ th.daaam.proceedings.126

\begin{abstract}
Exoskeletons are wearable devices that are widely used in various fields as; medicine, military, or industrial applications for human performance augmentation. They are highly complex systems and mostly used for specific purposes such as handling certain weights. Since this paper is concerned with the latter, therefore, a detailed literature review and industry research, with specific focus on relevant engineering standards has been conducted. The necessary software tools were chosen to complete a basic simulation of the working environment and initial design of an exoskeleton model. Relevant tool evaluations were conducted, in terms of motion studies and human-exoskeleton interaction analysis, on a theoretical aspect. The results of the conducted research, design, and simulations were successful, with a room for future improvement including several sections such as mechanical analysis or design improvements.
\end{abstract}

Keywords: Exoskeleton; Human Augmentation; Upper-body; Human-Machine Interaction

\section{Introduction}

This paper describes the research and development process of an upper body exoskeleton to be used as an industrial solution, as a form of human body performance augmentation, during handling medium loads (18-35 kg). It is comprised of research information on exoskeletons; in terms of industry, engineering standards and relevant literature review. In addition to developing a 3D exoskeleton model using SolidWorks, based on the obtained results from the research. The design is intended as an initial solution for lifting loads around $25 \mathrm{~kg}$ and to have flexibility for changes in load dimensions. Afterwards, the model is simulated using MATLAB's Simulink environment; to simulate the performance of suggested motors in the developed exoskeleton model and assess their results.

This work is initialized within the scope of a Mechatronics and Smart Systems Course Project in Tallinn University of Technology [1]. The preliminary direction was focused to find a general solution to avoid heavy lifting injuries and reduce work fatigue of a certain factory's workers. However, the direction of the project was changed to specifically wearable upper-body exoskeletons for production workers. This paper focuses on obtaining a basic mechanical design for the exoskeleton, along with performing the necessary simulation process to verify the obtained design. This, along with researching and adhering to engineering standards that govern these processes. 


\begin{tabular}{|c|c|c|c|}
\hline Intialization & Research & Development & Finalization \\
\hline $\begin{array}{l}\text { 1. Problem Description } \\
\text { 2. Team Building } \\
\text { 3. Preliminary Direction } \\
\text { 4. Solution Proposal } \\
\text { 5. Project Scope }\end{array}$ & $\begin{array}{l}\text { - Literature Review } \\
\text { - Industry Research } \\
\text { - Standards Definition } \\
\text { - Tools Evaluation }\end{array}$ & $\begin{array}{l}\text { - Model Design } \\
\text { - Motion Study } \\
\text { - Process Simulation } \\
\text { - Interaction Analysis } \\
\text { - Mechanical Analysis }\end{array}$ & $\begin{array}{l}\text { 1. Prototyping } \\
\text { 2. Testing } \\
\text { 3. Revising } \\
\text { 4. Producing }\end{array}$ \\
\hline
\end{tabular}

Fig. 1. Flowchart of the project steps

Figure 1 shows the flowchart of the project steps. Initialization and Finalization tasks have a certain order to follow for reaching the optimum result. However, Research and Development phases do not have an order in their respective subsections since these tasks should be done in parallel with getting constant feedback.

\section{Literature and Industry Review}

The definition of exoskeleton is an artificial external supporting structure [2]. They are wearable mobile machines, which are based on various combination of technologies like electro-mechanical, pneumatic or hydraulic. Exoskeletons are superior integrated human-robotic systems [3], whose main goal of development is human augmentation, or in simple words, physical improvement. They allow lifting more weight with more ease, or faster movement while handling loads.

Even though most studies on exoskeletons focus on very specific features, such as gait phase recognition, this research was performed in different fields [4]. Generally, an exoskeleton is a versatile device that can be used in various fields. Several devices and prototypes are developed for military, industry, medical and aerospace purposes. For example, industrial exoskeletons designed for supporting manual labour tasks in factories are now commercially available [5]. They are used for assistance in tasks like lifting and moving during logistical processes. They are considered an ergonomic solution for workers who perform physically demanding actions for long periods of time as; lifting heavy objects or simply standing up, which can significantly reduce stress on their bodies and enhance their performance.

\subsection{History of exoskeletons}

The earliest known exoskeleton-like device was a set of jumping and running assisted apparatus developed in 1890 in Russia. General Electric developed the first real exoskeleton device in the 1960s called Human Augmentation Research and Development Investigation or Hardiman in short [6]. It was a hydraulic and electrical bodysuit. However, it was too heavy and bulky [7]. The most significant projects related to this field were done between 1969-1972 in Serbia for medical assistance to disabled people. Moreover, the US Army is working on a military exoskeleton called LIFESUIT for soldiers' rehabilitation since 1986, which currently has 17 prototypes [8]. Pentagon even started to implement nanotechnology to exoskeleton projects in 2007. The latest major updates in this field for military purposes are performed by Exoskeletons for Human Performance Augmentation Program of The Defense Advanced Research Projects Agency (DARPA) [9].

\subsection{Exoskeleton types}

Exoskeletons can be divided in many ways. For example, according to their power consumption as powered (static or dynamic), passive (weight redistribution, energy capture, dampening, or locking), pseudo-passive, and hybridexoskeletons. In addition to that, they can be categorized according to their mobility as; fixed, supported or mobile. Another way to distinguish them is with their control method like joystick, sensors, mind-controlled, not-controlled, buttons or control panels. It is also possible to separate them according to if they are built with rigid or flexible materials. They can have different origins such as home-built, research labs, commercial companies, or governments [10]. However, the most common method of classification is by body parts; upper-body, lower-body and full-body exoskeletons.

\subsection{Human augmentation}

The field of human augmentation focuses on creating cognitive and physical enhancements in the human body [11]. When augmentation technologies applied, humans will be stronger, faster, and more productive. This is not only to compensate disabilities but also to drive human capacities beyond regular physiological levels by enabling to perform a diverse set of tasks [12]. Human augmentation is not only about exoskeleton. There are other ideas already in the market as a concept such as 3rd thumb [13], Duoskin tattoo to create customized functional devices attached directly on skin [14], airbag helmet made for mainly urban cyclists [15], and EnChroma glasses for people with colour blindness [16].

\subsection{Industry Research}

An example to upper-body exoskeleton companies is Exhauss, a French company that produces upper body exoskeletons for mid-level lifting. They are the first company to sell industrial exoskeletons, and their products can lift up to $25 \mathrm{~kg}$ [17]. Another example of an upper body exoskeleton is EksoVest; a mechanical upper-body exoskeleton that is operated using springs, and can support up to $6.8 \mathrm{~kg}$ per arm [18]. Various models of successful exoskeleton companies were investigated. Most findings were useful even though they were not particularly related to the project scope mostly because of their goals. Table 1 shows the commercial products of such upper-body exoskeleton companies. 


\begin{tabular}{|c|c|}
\hline Company & Products \\
\hline Atoun & Model-Y (10kg) [19], Model-A [20] \\
\hline Bioservo & Ironhand [21] \\
\hline EksoBionics & EksoVest (13kg) [18] \\
\hline Hyundai & H-WEX (60kg) [22] \\
\hline Levitate Technologies & Airframe [23] \\
\hline Panasonic & AWN-03 (15kg) [24] \\
\hline StrongArm Tech & V22 [25] \\
\hline SuitX & BackX [26], ShoulderX [27], MAX (legX + backX + shoulderX) [28] \\
\hline
\end{tabular}

Table 1. Commercial products of upper-body exoskeleton companies

However, there are several companies that produce very successful exoskeletons that is very similar to the project goals. Most significant ones were the upper body exoskeletons of Exhauss and Innophys with several options. Table 2 consists of most related products of these companies.

\begin{tabular}{|c|c|}
\hline Products of Exhauss & Products of Innophys \\
\hline Reliever (25kg) [29] & \\
Orbiter (17kg) [30] & Muscle Suit Power (35kg) [35] \\
Hanger (20kg) [31] & Muscle Suit Edge (25kg) [36] \\
Transporter (20kg) [32] & Muscle Upper (35kg) [37] \\
Worker (25kg) [33] & Muscle Suit (25kg) [38] \\
Lifter (25kg) [34] & \\
\hline
\end{tabular}

Table 2. Commercial products of Exhauss and Innophys

In addition to the discussed products and companies, the research resulted in many sources that proved beneficial, and were considered as references to improve the theoretical understanding on exoskeletons for practical purposes. These were considered as a source and used to improve the theoretical understanding on exoskeletons for practical purposes.

- $\quad$ European Space Agency: As an international organization with 22 members, European Space Agency (ESA) has an interest in developing exoskeletons for space robotics. ESA Human Arm Exoskeleton has been developed to enable in-space force-feedback telemanipulations with redundant robotic arms [39]. The target of this project is the application of astronaut-like robots' telemanipulation and operating in a remote harsh environment by robotuntrained operators placed in a comfortable (but gravity-less) environment.

- Nakamura Lab: The Biomechatronics lab of Chuo University, or Nakamura Lab, is working on projects related to biorobots and soft robotics. Related projects done by this lab are assistive devices focused on knee, leg, and waist. The waist assist project named AB-Wear has a similar research goal, removing back pain of people who lift heavy weights. The aim was to develop a flexible and light-weight assistive device with while considering safety [40].

- RotBot Systems: Founded in 2014 in Nes Ziona (Israel), they are the first to engage in the development of an industrial Exoskeleton based on a crowd sourcing module. The RotBot Exoskeleton suit is currently developed \& managed by Anthony Roth \& Sons Company in cooperation with IntoVision, RobotICan, and Robotics Laboratory Department of Mechanical Engineering of Ben Gurion University of the Negev [41]. This exoskeleton suit is mainly developed to augment strength to the suit wearer in industrial uses.

- Aalborg University: As well as developing AnyBody software and using it to develop passive and active exoskeleton systems, there are also several researches done about exoskeletons in Aalborg University of Denmark. For instance, the research paper on Design of a Passive Exoskeleton for the Upper Extremity through Co-simulation with a Biomechanical Human Arm Model is published by S. Bai, L. Zhou and J. Rasmussen. A simulation environment is developed to test human body mechanics and exoskeleton and to integrate them. As a result, 2 types of passive exoskeletons are designed for helping the patients [42]. In addition, a research was published on Design and Optimization of a Spring-loaded Cable-driven Robotic Exoskeleton where musculoskeletal human arm model is integrated with cable-driven exoskeleton model for simulation [43].

- MovAiD: A joint project of several companies under Horizon 2020 EU program. The aim of the program is to develop assistive technologies for elderly people and industry workers. The device measures the forces on the joints and angles of the joints by using many sensors. With the smartphone application the data can be read easily. Therefore, the total bio-feedback of the movements opens a way to identify the effects of the movements on human body [44].

- AXO-SUIT: A project includes 3 universities and 5 companies to build a full-body exoskeleton for elderly people. This medium-duty exoskeleton designed to lift and carry objects up to $5 \mathrm{~kg}$ for each arm. Design requirements were determined by a detailed survey including 34 people. According to results, AXO-SUIT designed to consist of 2 parts; a lower-body part (LB-AXO) and an upper-body part (UB-AXO) [45]. 
- Wearable Robotics Association: An organization aiming to connect all the developers and entrepreneurs around the world for the fast development of the wearable technologies. They organize conventions and conferences around the globe to bring all academics and businesses together every year [46]. One of their biggest events is WearRAcon which is organized every year to join all people who are contributing the developments in this field [47]. Also, the organization actively monitor the available wearable technologies and connect them to their network. The wearable robots archive of the organization is very wide. There are 7 different fields such as rehabilitation, manufacturing and construction, defence etc. [48].

- Exoskeleton Report: A non-profit and independent organization which is aimed to gather all the information from internet about wearable robotics and exoskeleton technologies. The website aims to become a center place for the information about all kinds of wearable robotics. They constantly monitor the developments in the technology and updates their website [49].

\subsection{Tools and Methods}

The tools used during this research include Dassault Systèmes' SolidWorks, to prepare the mechanical design of the exoskeleton and perform motion studies. Also, Visual Components software was used to prepare the process simulation of actual work.

Further results are obtained using Mathworks' MATLAB with Simulink environment, to simulate the appropriate motors to be used in the created exoskeleton design and assess their results. ANSYS software tools was selected for mechanical analysis that will be conducted in future works.

A well-developed software tool OpenSim was evaluated for human-machine interaction analysis. Further research showed that OpenSim is capable of many things needed in the project, but an exoskeleton analysis was never done before with this tool. The lack of previous research using this tool and the fact that the main goal of the tool was medical engineering forced the team to move on with another software. More on-point research showed that there is only one fully developed tool called AnyBody that has been used with several exoskeleton projects. AnyBody software was evaluated with a trial license to perform an interaction analysis of human body with the exoskeleton and it was agreed on to be used for the future of the project. The main reason of the choice was the availability of the previous similar works to review during the project.

\section{Standards and industry requirements}

Following a correct engineering design approach, a research was conducted to specify the relevant design and safety standards. Since exoskeletons are intended mainly as medical devices to be used in personal support and rehabilitation, the first encountered set of standards was relevant to medical applications; ISO 13485:2016 Medical devices - Quality management systems [50].

Further research revealed other standards related to exoskeletons within the scope of medical applications. The standards are known as "ISO 13482:2014 Robots and robotic devices - Safety requirements for personal care robots" [51] and they comprise the main part of this section. In addition to ISO standards, a separate set of standards that governs exoskeleton applications in industrial field was found. These standards are being developed by ASTM, Committee F48 [52] on Exoskeletons and Exosuits. However, this committee was recently established (2017), and the only published standards are relevant to terminology [53] and labelling [54].

This part of the paper will focus on explaining the most important sections of the current used standard; ISO 13482, which is implemented in almost all exoskeleton research studies.

\subsection{Terminology}

Physical assistant robots are personal care robots that physically assist a user to perform required tasks, by providing supplementation or augmentation of personal capabilities. They are classified into restraint type and restraint-free type physical assistant robots. Exoskeletons lie in the first category, defined as restraint-type physical assistant robot that is fastened to a human during usage [51].

\subsection{Mechanical Design}

Personal care robot shall be designed to reduce risks of hazards resulting from exposure to moving components such as motors, gears, wheels to an acceptable level. Design considerations include designing with a minimum number of accessible moving parts. Moreover, components such as motors, shafts, gears shall not be exposed.

According to ISO 12100, sharp edges and points shall be avoided in the design of a personal care robot. Holes or gaps shall be designed in a way that prevents insertion of any part of the human body, in compliance with ISO $13845 \&$ ISO 15534. Further safety measures shall be applied as; the use of form fitting designs, using passive means of securing loads (screws), limiting devices to avoid handling of loads that exceed its maximum rated payload.

As for physical stress, design of personal care robot shall consider its effect on its user due to continuous use. This is achieved by taking into consideration the typical body size of intended customer segments, to avoid physically demanding body postures. This is well-defined in ISO 14738, which describes how principles of ergonomic factors, by construction of workstations and machinery, should be applied. 
Moreover, a personal care robot user shall be protected from harmful vibrations that could cause vibration-related injuries; tendon inflammations, backache, neurosis, or similar injuries resulting from continuous use of the robot. This, as well as vibrations between $0.5 \mathrm{~Hz}$ and $80 \mathrm{~Hz}$ that can cause problems for health, comfort and perception, and vibrations between $0.1 \mathrm{~Hz}$ and $0.5 \mathrm{~Hz}$ that can cause motion sickness. Therefore, active vibration; through either semiactive damping mechanisms or control-based damping, or restriction of velocities of the personal care robot not to cause vibrations, control should be considered [51].

\subsection{Control}

During start-up, personal care robots shall perform internal control tests to ensure that availability of all safety-related functions. In case of failure to perform such test, it shall perform a protective stop. Moreover, personal care robot shall start up with restricted speed and force and shall return to normal state through a mode change. Other protective measures include startup only after feedback from sensors that no hazardous situations exist.

In case of automated decisions; inaccuracies can be reduced by increasing the decision's reliability (better sensors) or by limiting the effect of a wrong decision (narrow limits of use). Moreover, identification algorithms shall be designed in a way, where probabilities of correct decisions are calculated and measurable. Higher uncertainties shall be re-evaluated using alternative approaches and/or additional information. Decisions which can lead to hazardous situations shall go through validity checks [51].

\subsection{Electrical requirements}

Electrical equipment of a personal care robot is designed according to the relevant requirements of IEC 60204-1. All hazardous energy sources (electrical, mechanical, etc.) shall be isolated, and clearly identified. Any type of stored energy shall be kept as low as reasonably practicable. Due to the usage of batteries, the device is governed by clauses concerning uncontrolled release of stored energy, which applies in both operation and shutting down states, where the controlled release of stored energy shall not lead to any additional hazards. This includes adding guards or covers to minimize risks during release of energy and providing the robot with means to regulate its energy supply, to prevent overheating or overcurrents cause by overloads or short circuits.

For electrical equipment, it is recommended to use extra-low voltage sources according to IEC 61140 (below 25V AC and 60V DC). Other types of stored energy shall be kept as low as practicably possible.

As for static electricity, it is required to provide sufficient electrostatic discharge (ESD) protection that does not require any personal protective equipment from users. This, in addition to discharging any harmful electrostatic potential, and avoiding harmful malfunction due to ESD. This can be achieved by using conductive materials, discharging outer surfaces by earthing and using other techniques to prevent electrostatic charge build-up on touchable surfaces.

In cases of power failure and unintended shutdown of a personal care robot, devices shall be designed to ensure that risks due to dropped loads in the event of failure or shutdown of power, are acceptable. Moreover, if a part of a personal care robot presents a trapping hazard, means shall be provided to move such parts without drive power by a single person, taking in consideration complete range of potential users [51].

\section{Preliminary Design}

The obtained model using SolidWorks was inspired by many existing exoskeleton models. In the presented model, there are total of four rotational joints; the only movable joints in the design. Motors are added to move the joints in rotational direction. Since the model, shown in Figure 2, is an upper body exoskeleton, the four rotational joints are divided between two arms, i.e. two for left arm and two for right arm. For each arm, one motor is added to provide the motion of the elbow, and another for the shoulder. Both the elbow and shoulder can move in more than one degree of freedom. However, the exoskeleton was set to perform the process of lifting, therefore, only one degree of freedom was required for the motion of each joint.

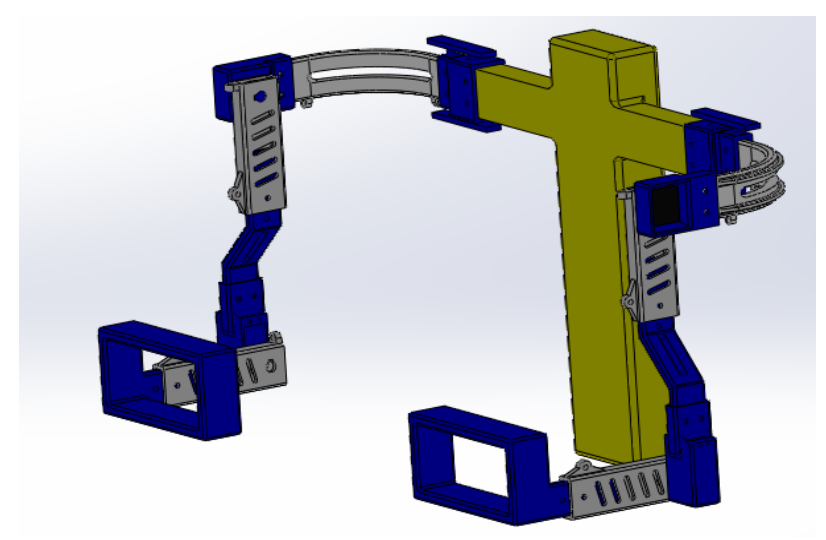

Fig. 2. 3D Design of the exoskeleton 
The model contains two fixed sliding translational joints, on each arm, which are adjusted according to the length of the user's arm. These translational joints were not taken into consideration in the motion analysis, as they are fixed during operation, however, they can be moved during the time of the apparatus setup, for adjusting purposes to specific users.

\subsection{Kinematics}

Kinematics of the human elbow can be mechanically represented as a single rotary joint. However, there exists a minor misalignment between the axes of the forearm and the upper arm. There is an angle between the two segments of the arm, and this angle is known as "carry angle" as shown in Figure 3 with a human skeleton model taken from AnyBody software [55]. This carry angle is adjusted by having the end of the exoskeleton arm holding the forearm only, excluding the hand, in addition to adding small freedom space, to enable the human to move the arm. This would make it possible to handle the item that will be lifted.

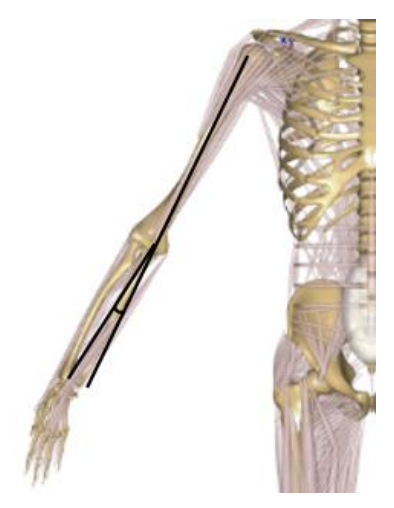

Fig. 3. Carry angle, shown with the model from AnyBody software

Motors' locations are already determined but can be altered or enhanced to fit any chosen motor with different dimensions. Modifications on the model are possible, if needed, however the current design is satisfactory. Moreover, the exoskeleton arms are mated to move parallel to each other, in order to avoid miscalculation of minimal imperfections in the mating process. Since a minimal imperfection would lead to the arms move in different paths with undesired angles, resulting in an inaccurate performance in real-life. Therefore, the parallel mate feature was used to make the simulations as close to real-life as possible.

\subsection{Hollow Parts}

Some of the exoskeleton parts have cut-offs; reduce the total mass of the exoskeleton and make it possible to introduce smaller motors for joints movement. The hollow parts can be changed according to a stress analysis of each part, and the application of maximum torque to study the bending moment. Implementations of this status can be seen in cut-offs of parts around the shoulder, connection parts between the joints, and parts to have translational joint for user's initial setup.

Adding the load to the exoskeleton shows the changes in the moment exerted on each part, which varies in each position the exoskeleton takes through its motion path as well. This can be studied carefully through a stress analysis of each part. Instead of performing a dynamic stress analysis, it is safer to apply maximum torque on each part, by adding the maximum load required in our case. This enables one end of the part to endure the load, and the other tip of the part will be fixed as cantilever joint. Moreover, adding a factor of safety to the design calculations, for a dynamic motion (Factor of safety =2), according to the standard set by Robert L. Mott in "Machine Elements in Mechanical Design" [56]. Even though this standard is made for machine elements, it would be safer for the operator to have a body that is designed to be as durable as machine elements, which are durable due to being expensive.

\subsection{Wire Routing}

Since the exoskeleton is an electromechanical device, therefore, an integration between electrical and mechanical components must take place. Design must take into consideration the required spaces to accommodate electrical components such as circuit boards, along with mechanical actuators. The wires along the arms of the exoskeleton must be long enough to handle the full cycle of the exoskeleton usage. Wires will also be tied using cable ties, to avoid strangle or hang up to other objects surrounding the body of the exoskeleton.

\section{Simulations}

Motion studies were conducted, using several software tools, to obtain a final simulation of the performance and working conditions of the developed exoskeleton model. Performed simulations are based on previous knowledge of SolidWorks motion studies, accompanied with MATLAB and Simulink modelling. An approximate simulation of the industrial work process was simulated using Visual Components software, however due to time limitations, it was conducted without the exoskeleton mounted on the human subject. 


\subsection{Model Simulation}

Using SolidWorks' motion study, a simulation of the normal motion performed by the exoskeleton during operation was performed. A load was added to the model, shown in Figure 4, to obtain results similar to those of the true work conditions. The study required each joint to perform a $90^{\circ}$ motion, from their initial position. The movement started from position No.1 to the final position No.2, as shown in Figure 4. Real life process does not require the exoskeleton to reach the final position shown in No.2, however, it was used during the simulation to obtain the maximum operating conditions of the proposed motors. This approach results in motor parameters that endure highest operation requirements and have longer durability while handling masses.
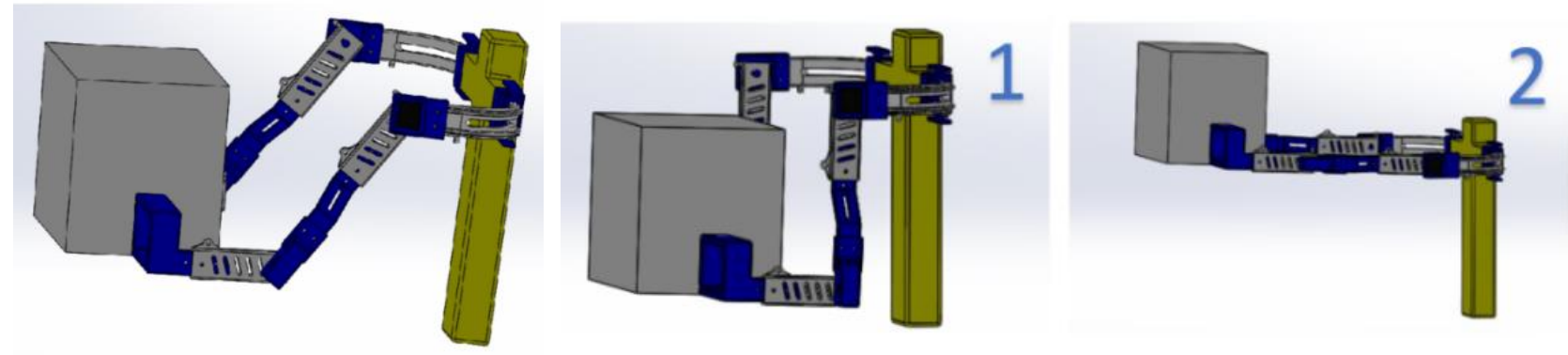

Fig. 4. Model with load and the positions of the motion

The 3D model was imported into the MATLAB environment, as a multi-domain model. Initially, the model is imported as in a static form with no motion. Motors were added to simulate the motion, since the previously added movements were not imported to the MATLAB simulation environment. Motor blocks were created and added to joints. During the motor selection process, three different motors from Maxon were compared, as Maxon motors are known to be used in several exoskeleton models. It was found that the most common motor type for upper-body exoskeletons were brushless DC motors. Therefore, three brushless DC motor models; EC-i 40 [57], EC 60 flat [58] and EC 90 [59] flat, were considered for this project. The most important parameters that affect the motor load are; stall torque (the torque that motor can produce with zero rotation), and no-load speed. The most appropriate motor, based on these specifications, was found to be EC-I 40, in addition to having suitable dimensions.

Simulation results show an in-rush current at the beginning of the simulation for each motor. This in-rush current can be harmful to the motor at high levels. However, in this case it does not exceeds the safe limits. Moreover, during physical implementation, a microcontroller can be added, which eliminates in-rush currents. After entering the parameters of ECI 40 motor for $18 \mathrm{~V}$ nominal voltage the first simulation is started and the model moved as expected as it can be seen in left side of Figure 5. Since there were no input to the motors in the beginning, arms of the exoskeleton fell downwards slowly because of the load attached to its hands. After the input voltage is given to all 4 motors, the arms started moving until they are horizontal. For the second simulation, the parameters for $48 \mathrm{~V}$ nominal voltage were entered. After the end of the simulation, it is observed that, with the same input given, the arms are moved more than needed. Therefore, input voltage level is lowered, and the expected result achieved as seen in right side of Figure 5.

1

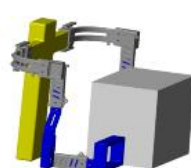

3

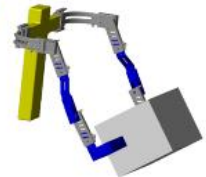

2

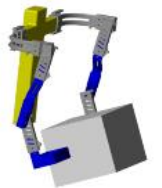

4

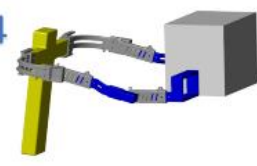

1

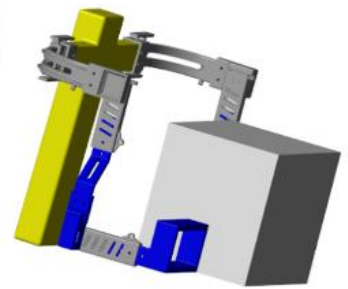

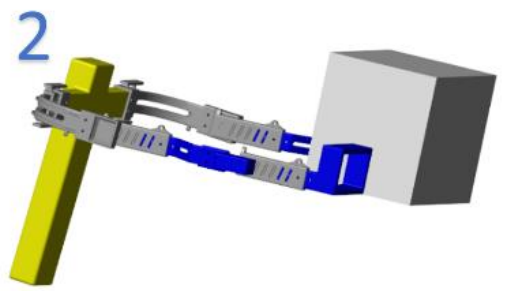

Fig. 5. Motion steps of model with motor parameters for $18 \mathrm{~V}$ and $48 \mathrm{~V}$ nominal voltage

\subsection{Interaction Simulation}

Any sort of assistive force or moments lead to contact forces with the human body. They depend on the exoskeleton design as well as the human behaviour. Basic information about the working process is necessary for the conceptual design. First, baseline analysis with no exoskeleton should be done to be used for subsequent comparison later. Full or estimated information about the motion of all body parts, joint angles, etc. should be known. 
It is essential to study machine's effect on human biomechanics like kinematics, moreover, muscle and joint reaction forces are a strong motivator. Estimation analyses of the interaction between the musculoskeletal system and the design of the exoskeleton is needed to achieve maximum effect and minimal discomfort. Another requirement is a cost-effective approach to predict and evaluate the important functionality of exoskeletons, e.g. maximum actuator force and precise alignment of exoskeleton joint locations with the anatomy of the user.

The current phase of the exoskeleton development process is pre-prototyping. In this phase, metabolic power play an important role in the design and evaluation, even though this phase does not end with a complete exoskeleton design. This phase comprises several steps; one of which is to add abstract forces and moments to body parts and joints and evaluate their effect on injury risks and performance bottlenecks. In addition to finding highly loaded muscles and joints to identify clever combinations of moments and forces that have the best effect as well as possible negative side effects.

Detailed design analysis has two major objectives. The first is kinematics analysis, concerned with kinematic compatibility between exoskeleton and human. This includes assessing the movement of the exoskeleton and collisions with the human body and choosing the actuator's range-of-motion. The Second is kinetics analysis which studies the contact forces between exoskeleton and human. Power consumption and peak power for actuators should be assessed. Peak forces/moments of actuators and metabolic power and energy consumption should be decided.

\subsection{Process Simulation}

The exoskeleton is designed for real-life lifting process. Therefore, the process simulated using Visual Components focused on the lifting action. The aim of this simulation is to be able to prepare a hardware-in-the-loop cycle when the project is applied to a real industrial plant in the future. The usage of the exoskeleton should cut down the cycle times by increasing the efficiency of the worker as well as decreasing the need of breaks by assisting the body. The simulation has many variables that can be altered after obtaining details as; manufacturer production process, items, etc. Moreover, the dimensions of all items used in the simulation could be changed to fit the real dimensions provided by the manufacturer.

The simulated process had a conveyor belt system that transports boxes, with masses of 18-35 kg, which are big enough to be held by human hands. At the end of the conveyor trip, a human picked up the box, and moved it to a pallet on the ground, and placed the box on that pallet. The boxes are stacked beside one another and over each other. The total number of boxes on the pallet was defined as 12 boxes, depending on the size of pallet and boxes in real-life conditions. The performed simulation is shown in Figure 6 below. The first figure is a visualization of picking up the box, followed by stopping to place the box, then placing it on the pallet, and the final arrangement of the full stack of twelve boxes.
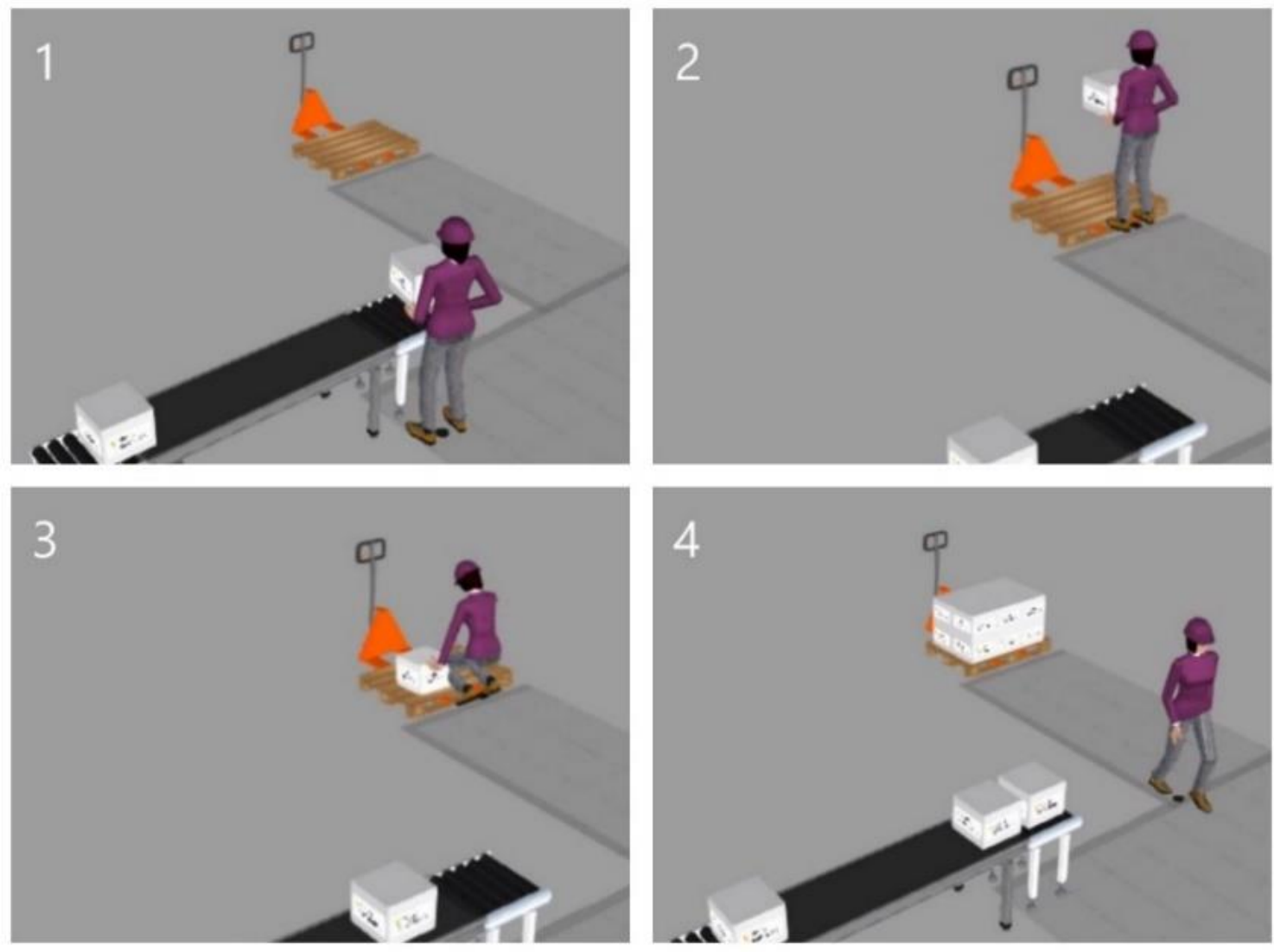

Fig. 6. Visualization of work process simulation 


\section{Conclusion}

As mentioned before, scopes of most exoskeleton studies are on very specific features, this work was concluded in various areas. The research on upper-body exoskeletons expanded to include several fields of applications; to reach the ground basis for all similar devices; design, electrical components, risks, etc. Moreover, an extended research in relevant engineering standards was conducted, to find what is applicable to exoskeletons. Given its nature, the applied standards included generic electrical and mechanical design standards, in addition to others that are specifically applied to exoskeletons in non-medical applications.

There are many aspects to consider while designing the exoskeleton such as safety standards, kinematic calculations, torque and forces applied, control, the human factor of hazard, actuators, transmission, effect of mass, material used, wire routing, and mechanical analyses that would include more specific details. The utilization of simulation software proved helpful to obtain a rough estimation of how the model should act in real-life. The used software tools facilitated the process of calculations, to estimate the path or trajectory of the model, as well as performing required analyses.

Since an exoskeleton is directly attached to the human body and can cause harm, safety is an important factor to consider during the design process. Not only software limitations must be set, but also mechanical switches are required to assure the safety of the exoskeleton. Throughout the design phase of the model, the assemblability and dismantlability of the exoskeleton were main concerns to be taken into consideration, in order to make it possible to implement in reallife as well as maintain the parts separately on long term. Taking these factors into consideration would guarantee saving time and money, otherwise, the dismantling process can cause failure in some parts of the exoskeleton.

The steps of project can be divided into four phases as it can be seen in the flow chart of the project. The Research phase was conducted in a very cautious attitude which resulted in a comprehensive review but cost a considerable amount of time. Currently the project is in the preliminary stages of the Development phase.

The process simulation comprised another aspect of the research phase of the presented project. The interaction analysis of the exoskeleton and human was completed on a theoretical level and it will be conducted in future works. The software was evaluated, and the best combination of tools was decided. This combination of software tools offers large potential for more detailed design results, provided enough time, budget, and a clear definition by any potential client. Mechanical analysis shall be completed as well as the detailed model design, and consequently new motion studies. Further steps in this section will include using the developed CAD model in the process simulation to obtain more accurate and complete process results. By the end of the research phase, which was incorporated with basic mechanical design, process simulation, and electrical component selection, the obtained results were very satisfactory, and offered a clear definition of the future direction of this project.

\section{Acknowledgments}

This work is supported by the Engineering and Information Technology faculties in Tallinn University of Technology. It has been concluded under the supervision of several professors and assistants. The most deserved acknowledgments are for Hakan Demir for assisting the project together with the team. Lastly, major acknowledgements are given to AnyBody Technologies for their guidance through the software evaluation period.

\section{References}

[1] Abdelmomen, M.; Demir, H.; Dengiz, O.; \& Samir, H. (2019). Research on Exoskeletons for Human Performance Augmentation: Wearable Upper-Body Exoskeleton for Production Workers -Tallinn University of Technology, Report, unpublished.

[2] https://www.merriamwebster.com, Definition of exoskeleton, Accessed on: 2019-05-10

[3] Kazerooni, H. (2008). Exoskeletons for Human Performance Augmentation. Springer Handbook of Robotics, $773-$ 793. DOI:10.1007/978-3-540-30301-5_34

[4] Broniszewski, M; Dabros, J; Iwaniec, M; Patyk M, \& Wesol, J (2017). Gait Phase Recognition for Exoskeleton Control Using Adaptive Neuro Fuzzy Inference System, Proceedings of the 28th DAAAM International Symposium, pp.0845-0853, B. Katalinic (Ed.), Published by DAAAM International, ISBN 978-3-902734-11-2, ISSN 1726-9679, Vienna, Austria DOI: 10.2507/28th.daaam.proceedings.118

[5] https://www.therobotreport.com/industrial-exoskeletons, (2018). The Robot Report, Industrial Exoskeletons: New Systems, Improved Technologies, Increasing Adoption, Accessed on: 2019-05-10

[6] http://cyberneticzoo.com (2010). Cybernetic Zoo, Man Amplifiers, 1965-71 - G.E. Hardiman I Exoskeleton - Ralph Mosher (American), Accessed on: 2019-05-10

[7] https://www.thoughtco.com, (2017). ThoughtCo, Exoskeleton, Accessed on: 2019-05-10

[8] http://www.theyshallwalk.org. They Shall Walk, Timeline, Accessed on: 2019-05-10

[9] https://www.science.gov. Science.gov, Sample Records for Darpa Exoskeleton Program, Accessed on: 2019-05-10

[10] https://exoskeletonreport.com, (2015). Exoskeleton Report, Types and Classifications of Exoskeletons, Accessed on: 2019-05-12

[11] https://www.gartner.com. Gartner IT Glossary, Human Augmentation, Accessed on: 2019-05-10 
[12] https://www.sciencemag.org, (2018). American Association for the Advancement of Science, Robotics for Human Augmentation. Accessed on: 2019-03-02

[13] https://www.daniclodedesign.com. Danielle Clode Design, The Third Thumb. Accessed on: 2019-05-12

[14] Kao, H. C.; Holz, C.; Roseway, A.; Calvo, A. \& Schmandt C. (2016). DuoSkin: Rapidly Prototyping On-Skin User Interfaces Using Skin-Friendly Materials. Available from: https://duoskin.media.mit.edu/duoskin_iswc16.pdf Accessed: 2019-03-02

[15] https://hovding.com. Hovding, Airbag For Urban Cyclists, Accessed on: 2019-03-02

[16] https://enchroma.com, (2018). EnChroma, How EnChroma Glasses Work, Accessed on: 2019-03-02

[17] http://www.exhauss.com. Exhauss, Exhauss System, Accessed on: 2019-03-05

[18] https://eksobionics.com. EksoBionics, EksoVest, Accessed on: 2019-03-05

[19] http://atoun.co.jp. Atoun, Model Y, Accessed on: 2019-03-05

[20] http://atoun.co.jp. Atoun, Model A, Accessed on: 2019-03-05

[21] https://www.bioservo.com. BioServo, Ironhand, Accessed on: 2019-03-05

[22] https://exoskeletonreport.com. Exoskeleton Report, Hyundai H-WEX, Accessed on: 2019-03-05

[23] https://www.levitatetech.com. Levitate Technologies, Airframe, Accessed on: 2019-03-05

[24] https://exoskeletonreport.com. Exoskeleton Report, Panasonic AWN-03, Accessed on: 2019-03-05

[25] https://www.strongarmtech.com. StrongArm Technologies, V22, Accessed on: 2019-03-05

[26] https://www.suitx.com. SuitX, BackX, Accessed on: 2019-03-05

[27] https://www.suitx.com. SuitX, ShoulderX, Accessed on: 2019-03-05

[28] https://www.suitx.com. SuitX, MAX (Modular Agile eXoskeleton), Accessed on: 2019-03-05

[29] http://www.exhauss.com, (2018). Exhauss System, Reliever, Accessed on: 2019-03-05

[30] http://www.exhauss.com, (2018). Exhauss System, Orbiter, Accessed on: 2019-03-05

[31] http://www.exhauss.com, (2018). Exhauss System, Hanger, Accessed on: 2019-03-05

[32] http://www.exhauss.com, (2018). Exhauss System, Transporter, Accessed on: 2019-03-05

[33] http://www.exhauss.com, (2018). Exhauss System, Worker, Accessed on: 2019-03-05

[34] http://www.exhauss.com, (2018). Exhauss System, Lifter, Accessed on: 2019-03-05

[35] https://innophys.jp. Innophys, Muscle Suit Power, マッスルスーッ Power, Accessed on: 2019-03-05

[36] https://innophys.jp. Innophys, Muscle Suit Edge, マッスルスーツ Edge, Accessed on: 2019-03-05

[37] https://innophys.jp. Innophys, Upper Muscle, マッスルアッパー, Accessed on: 2019-03-05

[38] https://innophys.jp. Innophys, Muscle suit, マッスルスーツ, Accessed on: 2019-03-05

[39] Vaibhav, A. \& Atul, D. (2010). Application of Exoskeleton for Space Robotics, Proceedings of INTERACT-2010, Chennai, ISBN: 978-1-4244-9006-6, pp. 298-302, IEEE, . DOI: 10.1109/INTERACT.2010.5706165

[40] http://www.mech.chuo-u.ac.jp. Nakamura Lab, Waist assist, 腰アシスト, Accessed on: 2019-03-05

[41] https://www.rot-bot.com. RotBot Systems, Accessed on: 2019-03-05

[42] Zhou, L.; Bai, S. \& Rasmussen, J. (2013). Design of a Passive Exoskeleton for the Upper Extremity through Cosimulation with a Biomechanical Human Arm Model, Proceedings of 1st AAU Workshop on Human-Centered Robotics, Aalborg, ISBN: 978-87-7112-090-5, Bai, S.; Larsen J.; Madsen, O. \& Rehm, M. (Eds.), pp. 8-14, Aalborg Universitetsforlag, Aalborg.

[43] Zhou, L.; Bai, S.; Andersen, M. \& John Rasmussen. (2012). Design and Optimization of a Spring-loaded Cabledriven Robotic Exoskeleton, Proceedings of 25th Nordic Seminar on Computational Mechanics, ISSN: 0281-6679, ISBN: 978-91-7473-456-0, Lund, Denmark.

[44] Gupta, P. (2015). MovAid- A Novel Device for Advanced Rehabilitation Monitoring, Proceedings of 37th Annual International Conference of the IEEE Engineering in Medicine and Biology Society, Milan, ISSN: 1558-4615, ISBN: 978-1-4244-9271-8, pp. 4655-4658, IEEE, DOI: 10.1109/EMBC.2015.7319432.

[45] https://www.axo-suit.eu. Axo-Suit, Accessed on: 2019-03-05

[46] http://www.wearablerobotics.com. Wearable Robotics Association, Accessed on: 2019-03-05

[47] http://www.wearablerobotics.com. Wearable Robotics Association, WearRAcon 19, Accessed on: 2019-03-05

[48] http://www.wearablerobotics.com. Wearable Robotics Association, Wearable Robots, Accessed on: 2019-05-10

[49] https://exoskeletonreport.com, (2018). Exoskeleton Report, Accessed on: 2019-05-10

[50] https://www.iso.org, (2016). International Organization for Standardization, Medical devices -- Quality management systems -- Requirements for regulatory purposes, Accessed on: 2019-05-10

[51] https://www.iso.org, (2014). International Organization for Standardization, Robots and robotic devices -- Safety requirements for personal care robots, Accessed on: 2019-05-11

[52] https://www.astm.org, (2017). ASTM International, Committee F48 on Exoskeletons and Exosuits, Accessed on: 2019-05-10

[53] https://www.astm.org, (2019). ASTM International, Standard Terminology for Exoskeletons and Exosuits1 Active Standard, Accessed on: 2019-05-11

[54] https://www.astm.org, (2018). ASTM International, Standard Practice for Labeling and Information for Exoskeletons, Accessed on: 2019-05-10

[55] Liszka, M S. (2016). Mechanical Design of a Robotic Arm Exoskeleton for Shoulder Rehabilitation, M.Sc. Dissertation, Department of Aerospace Engineering, University of Maryland, Maryland, United States.

[56] Mott, R. L. (2004). Machine Elements in Mechanical Design, Pearson, ISBN: 0-13-061885-3, New Jersey.

[57] https://www.maxonmotor.com. Maxon, Motor, EC-i 40, Accessed on: 2019-05-12

[58] https://www.maxonmotor.com. Maxon, Motor, EC 60 Flat, Accessed on: 2019-05-10

[59] https://www.maxonmotor.com. Maxon, Motor, EC 90 Flat, Accessed on: 2019-05-10 